\title{
Printing Dynamics: Nip Pressure and Its Relationship with Materials' Viscoelasticity
}

\author{
Li Yang ${ }^{1}$
}

Received: 22 January 2020 / Accepted: 8 April 2020 / Published online: 21 April 2020

(c) The Author(s) 2020

\begin{abstract}
A theoretical framework of nip dynamics of conventional printing, including dynamic models deducted from nip geometry, printing speed, and physics laws, is proposed. Different from previous works, the present work focuses at obtaining the nip pressure from a given nip geometric setting, the common way in full-scale printing. The effects of viscoelastic characteristics of paper substrate and print form (rubber and/or polymer) on the nip pressure, which become pronounced in a full-scale printing process due to high speed, are accounted and illustrated by three physical models, e.g., Maxwell model, Kelvin-Voigt model, and Burgers model. Details of the nip dynamic features, shape, amplitude, duration, and effective nip width, etc., have been worked out. The viscoelastic nature of the materials was found to be responsible for the so-called speed-hardening, asymmetric nip profile, variations in the nip amplitude and effective nip width, etc. It was also found that how the viscoelastic properties of the materials affect the nip dynamics depend on the how the elastic components and the viscos count parts are connected with each other. The framework is applicable to calendaring, gravure, offset, and flexography.
\end{abstract}

Keywords Printing dynamics $\cdot$ Viscoelasticity $\cdot$ Board compressibility $\cdot$ Nip pressure $\cdot$ Packaging

\section{Introduction}

In conventional printing techniques, e.g., offset and flexography, ink transferring from a print nip to a paper (board) surface relies on mechanical contact between the print form (nip) and the paper surface [1]. To promote the contact a nip pressure is applied. Under the nip pressure, both the print form and the board undergo dynamic compressions and deformations which improve the contacts between the print form and the board surface and even out surface topographic variations. Thanks to the nip pressure, ink is partially pressed into pore structure of the paper surface [2].

Detail nip-pressure profile, i.e., shape and width or time duration of the nip pressure, governs the ink transferring thereby print quality. However, in full-scale printing, nip pressure is never directly set. Rather, it is the gap between the plate cylinder and the impression cylinder that is set. Prior to a production run, the nip is brought to a so-called "barely touch" position at first, where the printing form

Li Yang

li.yang@ri.se

1 RISE-Research Institutes of Sweden, Drottning Kristinas väg 61, 11486 Stockholm, Sweden "kisses" the paper surface. Then the gap is incrementally reduced to increase the nip pressure. Following each gap reduction, a short printing run is conducted and print quality reviewed. When the print quality is approved the nip setting (the gap) is fixed for the production run. In other words, no explicit nip pressure value is targeted in the nip-setting process.

In papermaking industry, compressibility of paper product is measured by the PPS method [3]. It is calculated from two PPS roughness values measured at two clamping pressures, namely:

$K=\frac{100\left(G_{1}-G_{2}\right)}{G_{1}}$,

where $G_{1}$ and $G_{2}$ are the PPS roughness values obtained at the nominal clamping pressures 1 and $2 \mathrm{MPa}$, respectively.

The PPS compressibility has intrinsic weaknesses when used to study a printing process. Even though the quantity obtained is named as Print-Surf compressibility, it actually measures the static compressibility, given a sufficiently long time for the board to deform under the pressure loads. This differs significantly from what occurs at a print nip where the dwelling time is very brief. A typical flexographic printer runs at a speed of $300-600 \mathrm{~m} / \mathrm{min}$. The corresponding nip 
time when nip compression occurs ranges in a few milliseconds (ms). Hence, to understand a print process, one needs to measure the dynamic compressibility of the board.

Holmvall and Uesaka [4-7] published a number of works on nip mechanics in flexographic post-printing on corrugated boards. A finite-element model of flexography was developed, and the material and geometrical properties of the system investigated. In their model, all materials were assumed elastic [4]. The results showed that it was the photopolymer and the top liner that had the biggest impact on the printing pressure variations.

Dubé and co-workers studied the hydrodynamic flow of a half-tone dot pressed onto a model porous substrate by a nip that moved vertically either close to or away from the substrate surface [8]. Ink movement under this nip pressure was described by the lubrication approximation of the Navier-Stokes equations taking into account of fluid tack. The transfer of a fluid dot onto a two-dimensional network of pores was simulated employing a "diffuse interface" technique. It was found that, at constant porosity, fluid transfer increases with decreasing pore size.

Jurkiewicz and Pyryev [9] studied the roller compression of the inking unit in an offset printing machine, wherein the ink roller (rubber-covered) was compressed against the plate roller (metal). In the proposed model, the materials properties were described by the Young's modulus and Poisson's ratio of the rollers' materials. The expression for the contact area width (nip width) was worked out, depending on the roller's geometry, the rollers materials properties and the peak clamping pressure. As no time aspect of roller compression was considered, the model does not account for the dynamic compression occurring in a true offset printing process.

Paper is a viscoelastic material [10], so does the rubber and polymers [11]. The viscoelastic nature of the materials can cause variability in test data and interpretation of test data requires an understanding of elasticity, viscoelasticity, stress strain (elastic modulus), etc.[10]. From a mechanics point of view, the mechanical response of a viscoelastic deformation is linearly proportional to the rate of deformation. The effect of viscoelasticity becomes thus more pronounced when the rate of deformation is high, as it is the case in full-scale printing. For example, the dynamic compressive strength of the soft materials, e.g., clay and sorbothane, increased by 4 and 3 orders of magnitude compared to their respective static values [12]. Hence, to be able to understand what happens in a printing nip of full-scale printing, techniques that enable one to measure both elastic and viscos characteristics of the materials involved in printing are needed [13].

While it is generally true that nip pressure varies with the gap of the nip, nip-paper interaction, or nip pressure is not solely defined by the nip geometry and the elastic properties
(Young modulus) of the materials involved, e.g., print form and paper. The viscoelastic nature rather than pure elastic property of the materials becomes important when printing speed is high, which has often been overlooked. In elastic deformation, the stress is proportional to the strain and independent of the rate of the strain, while in viscos deformation, the stress is proportional of the rate of the strain rather than the strain itself. The latter is probably responsible for the so-called speed-hardening, as one will see later in this article.

Creep is the deformation of a material occurring with time due to an externally applied constant stress. For cushioning materials specifically, it may be defined as the change in thickness of a cushion under static compressive load over a period of time [14]. It is also referenced as a procedure designed to determine the resistance of a paper board to a vertically applied constant load for a specified time $[15,16]$. In practice, the time of failure can range from less than $1 \mathrm{~h}$ to decades, with lower applied loads resulting in slower deformation and longer box lifetimes (time to failure from initial loading) [17].

There are a number of material models that have often been used in studying viscoelastic materials, e.g., rubber and polymer, etc.[18]. The basic components of the models are spring(s) and dashpot(s), representing elastic and viscos deformations, which are in series or parallel connections or in combinations [19]. The constituent in most paper is pulp fiber which contains pure cellulose, hemicellulose, and lignin. Since all these three components are polymers, i.e., consist of long molecular chains or large networks of covalently bonded atoms, the precedingly mentioned theoretical models had naturally be applied to paper-based materials for above all studying of creep phenomena [11]. These models or their variants have also been used to study calendaring [20] and mechanical characterization of soft materials [12]. It has been found, among other things, that viscoelasticity of the paper is responsible for the shift of the peak nip pressure from the geometric nip center towards the nip entrance [20].

Here, we present a theoretical approach to printing dynamics with a special focus on nip pressure that a paperboard experiences inside the printing nip. The expression for the nip pressure as a function of viscoelastic characteristics of the materials involved, e.g. paperboard, rubber blanket, and polymer plate, etc., in addition to the geometrical settings, printing speed etc., has been worked out. The approach is demonstrated by employing the material models to a number of practical applications.

\section{Model of Paper Deformation in the Printing Nip}

In practice, a printer is set by adjusting the nip opening which is the minimal gap between the nip cylinders rather than nip pressure. The nip pressure is the consequence of the 
nip-paper interactions, depending on the materials properties of the print form and the paper, the nip opening, the initial paper thickness, and the printing speed. Hence, it is necessary to work out the model of paper deformation when it goes through a printing nip, prior to introducing the printing dynamics models.

Figure 1 (left) illustrates the interactions in the printer nip between the print form and the paper substrate. For a given nip opening and an initial paper thickness, the nip pressure and the nip width may still vary with the dynamic compressibility of the paper substrate, represented by the paper's viscoelastic characteristics, as one will see later in this work. Hence, for convenience of modeling without losing generality, we assume that the nip rollers are two rigid cylinders, while the print form together with the substrate forms the material stack which passes through the opening between these rigid cylinders, as shown in Fig. 1 (left). An obvious advantage of such treatment is its general applicability. It is directly applicable to gravure when the print form is omitted in the material stack. It also applies to either offset or flexography when different print form (rubber or polymer) is involved. Furthermore, it applies even to calendaring with hard or soft nip.

Figure 2 shows the nip geometry of a printing press consisting of two rigid cylinders. The radii of the cylinders are $R_{1}$ and $R_{2}$, respectively. The speed of the material stack going through the nip is $v$. When going through the printing nip, the material stack experiences nip compression in three major steps, e.g., (1) initial deformation as the stack enters the nip at $t=0$ and the nip pressure increases with time; (2) maximal deformation while the stack approaches and passes through the nip center at $t=t_{\mathrm{c}}$; (3) recovering after the nip center and the nip pressure decreases with time

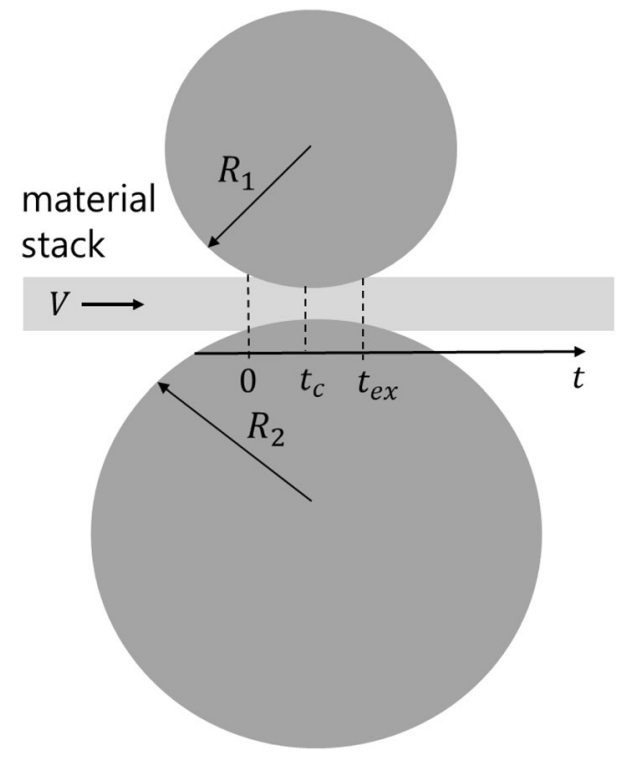

Fig. 2 Illustration of the nip geometry and the time when the paper sheet exits the nip

and completely vanishes when the material stack exits the nip at $t=t_{\mathrm{ex}}$.

Assuming that the initial thickness of the material stack is $D_{0}$ and the minimal gap between the cylinders $g_{0}$, the thickness of the material stack in the nip can be expressed as:

$$
\begin{aligned}
D(t) & =g_{0}+\frac{v^{2}\left(t-t_{\mathrm{c}}\right)^{2}}{2}\left(\frac{1}{R_{1}}+\frac{1}{R_{2}}\right) \\
& =g_{0}+\emptyset\left(t-t_{\mathrm{c}}\right)^{2}, \quad\left(0 \leq t \leq t_{\mathrm{ex}}\right),
\end{aligned}
$$

Fig. 1 Illustration of the proposed scheme wherein the print form is treated as part of the material stack together with the paper substrate forming a sandwich type of material structure (left); the interaction of a printing nip with the paper substrate is also shown on the right
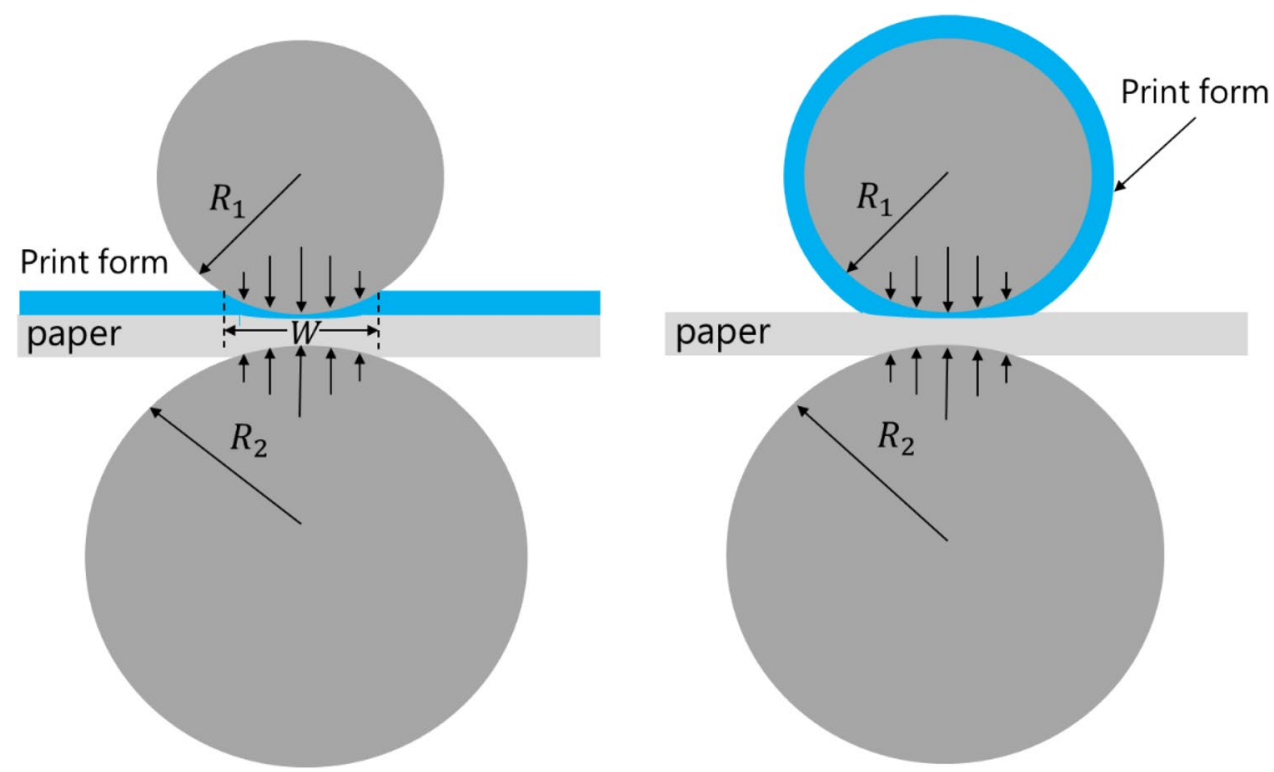
where the quantity:

$\emptyset=\frac{v^{2}}{2}\left(\frac{1}{R_{1}}+\frac{1}{R_{2}}\right)$.

The time interval from entering the nip to reach the nip center equals:

$t_{\mathrm{c}}=\sqrt{\frac{2\left(D_{0}-g_{0}\right) R_{1} R_{2}}{v^{2}\left(R_{1}+R_{2}\right)}}=\frac{W_{0}}{2 v}$,

where

$W_{0}=\sqrt{\frac{8\left(D_{0}-g_{0}\right) R_{1} R_{2}}{R_{1}+R_{2}}}$,

is the nominal nip width corresponding to the length from where the material stack enters the nip to where it exits, provided that the material stack fully recovers in thickness when it exits the nip. In such a case, the nominal nip width depends solely on the geometries of the nip cylinders, the nip opening, and the initial thickness of the material stack.

The strain of the material stack equals:

$\varepsilon=\frac{D(t)-D_{0}}{D_{0}}=\varepsilon_{0}+\frac{\emptyset}{D_{0}}\left(t-t_{\mathrm{c}}\right)^{2}, \quad\left(0 \leq t \leq t_{\mathrm{ex}}\right)$,

where the quantity

$\varepsilon_{0}=-1+\frac{g_{0}}{D_{0}}$.

\section{Models of Dynamic Compression of the Material Stack}

In the print nip, the paper is subjected to compressive stress of the print nip and undergoes conformational changes and by viscoelastic flow. To analyze the viscoelastic response in creep and relaxation experiments, spring and dashpot elements are frequently used [21,22]. The spring stretches or compresses instantaneously under the stress, whereas the plunger immersed in the dashpot filled with a Newtonian liquid moves at a rate proportional to the stress. Mathematically, the ideal spring obeys Hooke's law:

$\sigma_{\mathrm{e}}=E \cdot \varepsilon$,

while the liquid in the dashpot obeys Newton's law of viscosity:

$\sigma_{\mathrm{v}}=\eta \cdot \dot{\varepsilon}$
Consulting Eq. (5), one can easily see that the compressive rate is linearly proportional to the printing speed:

$\dot{\varepsilon} \propto v$.

Hence, it is necessary to properly deal with viscos behavior of the paper and print form (rubber or polymer) in addition to their elasticities, as it depends strongly on the printing speed.

In this section, we revisit three well-known material models which had been used in studying creeping or relaxation behavior. The existing studies focused on finding deformation behavior with respect to a known loading condition (stress), while the present study focuses on finding the nip pressure (the stress) when the deformation of the material is known or defined by the nip geometry. Another difference is the speed of deformation as the material undergoes rapid deformation amid to high printing speed, up to $10 \mathrm{~m} \mathrm{~s}^{-1}$ in a full-scale flexographic printing.

It is worthy of noticing that paperboard is regarded as a basic building block in this framework, even though it could be of multiply or in layered structure. In addition, even though the Young's modulus in Eq. (7) is treated as constant in the calculations, the proposed framework can easily be extended to situations where the Young's modulus is not constant. Strategy to cope with situations of non-constant Young's modulus is presented in the section Discussion.

\section{Maxwell Model}

The Maxwell model consists of a Hookean spring and a Newtonian dashpot connected in series [21], as shown in Fig. 3. The spring should be visualized as representing the elastic or energetic component of the response, while the dashpot represents the conformational or entropic component. In a series connection, the stress on each element is the same and equal to the imposed stress, while the total strain is the sum of the strains of each of the elements. Hence, there are:

$\sigma=E \cdot \varepsilon_{1}=\eta \cdot \dot{\varepsilon}_{2}$,

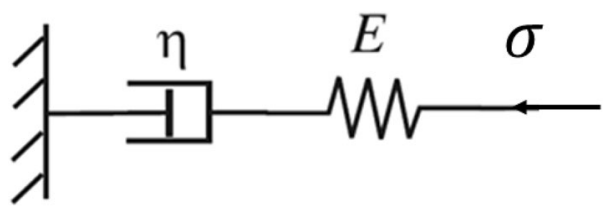

Fig. 3 Schematic representation of Maxwell model 
where $\varepsilon_{1}$ and $\varepsilon_{2}$ are the strains of the spring and the damper and the total strain is the sum of them, $\varepsilon=\varepsilon_{1}+\varepsilon_{2}$. The model can be represented by the following equation:

$\dot{\sigma}+\frac{E}{\eta} \sigma=E \dot{\varepsilon}$.

Replace the total strain with its expression given in Eq. 3, one receives the following equation:

$\dot{\sigma}+\frac{E}{\eta} \sigma=2 \frac{E \cdot \emptyset}{D_{0}}\left(t-t_{\mathrm{c}}\right)$,

which describes the time variation of the nip pressure (stress) in the printing nip.

The solution of this differential equation is:

$\sigma(t)=2 \frac{\eta \cdot \emptyset}{D_{0}} t+2 \frac{\eta \cdot \emptyset}{D_{0}}\left(t_{\mathrm{c}}+\frac{\eta}{E}\right)\left[\exp \left(-\frac{E}{\eta} t\right)-1\right]$,

provided that the initial stress at $t=0$ when the material stack enters the nip is $\sigma_{0}=0$.

\section{Kelvin-Voigt Model}

The Kelvin-Voigt $(\mathrm{K}-\mathrm{V})$ model consists of a purely viscous damper and a purely elastic spring connected in parallel, as shown in Fig. 4, which is often used in studying creeping behavior of polymers. The mathematical expression of the model is:

$\sigma=E \cdot \varepsilon+\eta \cdot \dot{\varepsilon}$.

Replacing the strain with its expression given in Eq. (3), the nip pressure can be expressed as:

$\sigma=E\left[-1+\frac{g_{0}}{D_{0}}+\frac{\emptyset}{D_{0}}\left(t-t_{\mathrm{c}}\right)^{2}\right]+2 \eta \frac{\emptyset}{D_{0}}\left(t-t_{\mathrm{c}}\right)$.

It is worth to notice that the second term in the equation, corresponding to the viscos component, is non-zero

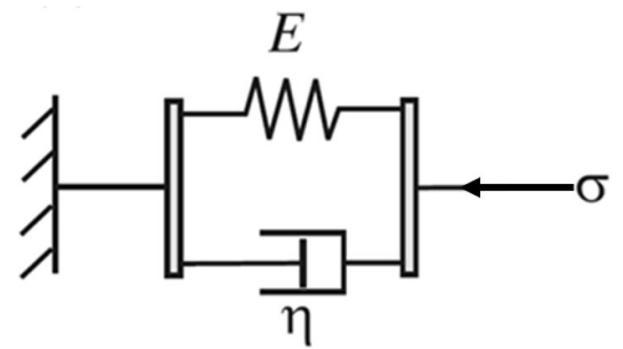

Fig. 4 Schematic representation of Kelvin-Voigt model at $t=0$. It means that there is a sudden increase in the nip pressure when the material stack enters the nip, that is:

$\sigma_{0}=-\eta \frac{V^{2}}{D_{0}}\left(\frac{1}{R_{1}}+\frac{1}{R_{2}}\right) t_{\mathrm{c}} \cong-\eta \frac{V \cdot W}{2 D_{0}}\left(\frac{1}{R_{1}}+\frac{1}{R_{2}}\right)$,

provided that $t_{\mathrm{ex}}=2 t_{\mathrm{c}}$, then $W=2 \mathrm{~V} \cdot t_{\mathrm{c}}$. The minus sign in the expression means that it is a compressive stress.

\section{Burgers Model}

Burgers model combines Maxwell model with Kelvin-Voigt model, which represents a sandwich type of material system, e.g., a Maxwell material and a Kelvin-Voigt material in series, as shown in Fig. 5. The mathematical expression for the model is:

$A \cdot \ddot{\sigma}+B \cdot \dot{\sigma}+\sigma=D \cdot \dot{\varepsilon}+E \ddot{\varepsilon}$,

where

$A=\frac{\eta_{1} \eta_{2}}{E_{1} E_{2}}, B=\left(\frac{\eta_{1}}{E_{1}}+\frac{\eta_{2}}{E_{1}}+\frac{\eta_{2}}{E_{2}}\right), D=\eta_{2}, E=\frac{\eta_{1} \eta_{2}}{E_{1}}$.

The general solution of the equation Eq. (17) is:

$\sigma(t)=C_{1} \mathrm{e}^{r_{+} t}+C_{2} \mathrm{e}^{r_{-} t}+C_{3}\left(t-t_{\mathrm{c}}\right)+C_{4}$,

where

$r_{ \pm}=\frac{-B \pm \sqrt{B^{2}-4 A}}{2 A}$.

As worked out in the Appendix, the four unknowns of the general solution given in Eq. (19) can be obtained by solving the linear equations:

$\left(\begin{array}{cccc}1 & 1 & -t_{\mathrm{c}} & 1 \\ r_{+} & r_{-} & 1 & 0 \\ 0 & 0 & 1 & 0 \\ 0 & 0 & B & 1\end{array}\right)\left(\begin{array}{l}C_{1} \\ C_{2} \\ C_{3} \\ C_{4}\end{array}\right)=\frac{2 \varnothing}{D_{0}}\left(\begin{array}{c}0 \\ -E_{2} t_{\mathrm{c}} \\ D \\ E\end{array}\right)$.

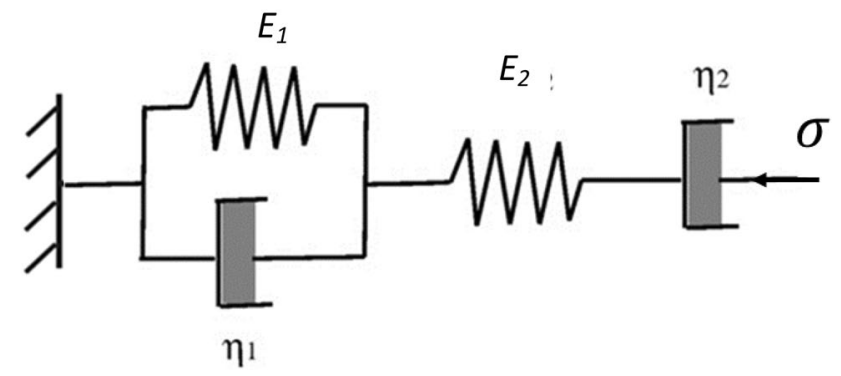

Fig. 5 Illustration of Burgers model 


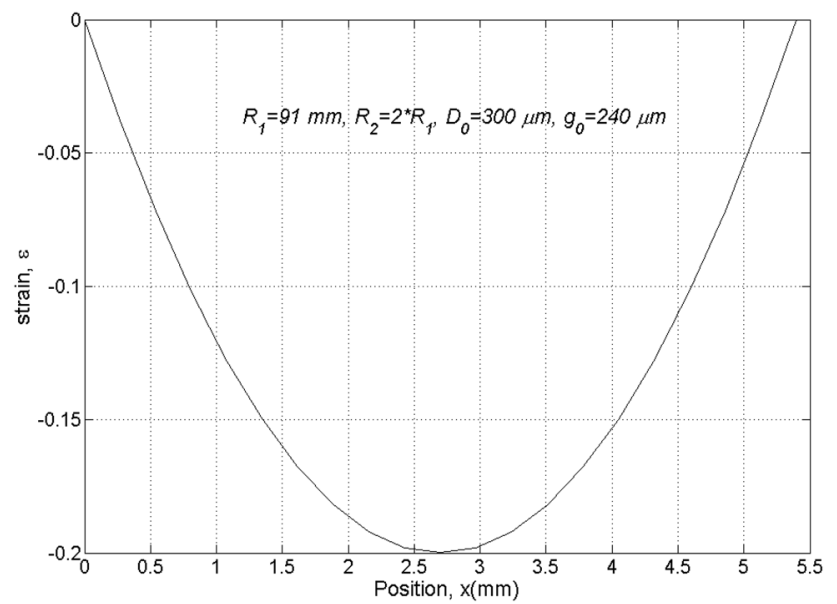

Fig. 6 Evolution of strain of the material stack that goes through the printer nip from entering the nip at $x=0$ to the nip center $(x=2.7 \mathrm{~mm})$ and to exiting the nip at $x=5.4 \mathrm{~mm}$

\section{Results}

The models presented in the proceeding sections can be used to study the dynamics of printing and calendaring processes. The focus will be given to the dynamic interactions between the nip and the material stack that passes through the nip. These dynamic interactions result in different nip pressures depending on the material characteristics and printing speed, e.g., combinations of material's elasticity with its viscosity. In printing processes, nip pressure plays a dominant role in ink transferring. Under the nip pressure, ink is pressed either into the substrate pore structure or aside on the paper surface, which dominates the outcome of both printing and calendaring.

For an easy appreciation of the results, the same parameters of the printing press are used throughout this work, namely, $R_{1}=91 \mathrm{~mm}, R_{2}=2 R_{1}$, and $v=10 \mathrm{~m} \mathrm{~s}^{-1}$, unless specially given. The thicknesses of the materials used in the simulations are $300 \mathrm{um}$ for paperboard and $2 \mathrm{~mm}$ for print form (rubber or polymer).

\section{Nominal Nip Profile}

Figure 6 depicts thickness evaluation of the material stack (paperboard) in the nip. The material stack enters the nip between the cylinders from the left $(x=0)$ and exits from the right. The distance from the entering position to the exiting is called the nominal nip width, $W_{0}=5.4 \mathrm{~mm}$, provided that the material regains its initial thickness $(300 \mu \mathrm{m})$ when it exits the nip. The geometries of the cylinders used in the simulations are, $R_{1}=91 \mathrm{~mm}$ and $R_{2}=2 R_{1}$, respectively, and the minimum gap between them is $g_{0}=240 \mathrm{um}$. However, one has to bear in mind that true nip width may differ from its nominal, as it depends on the material characteristics and printing speed.

When passing through the nip, the material undergoes rapid deformation mainly in $Z$ direction in addition to shear in $x-y$-plane [23]. The material first experiences compressive deformation before reaching the geometric center of the nip. After the nip center, the material recovers. When exiting the nip, the material has recovered either partially or fully from the compression, depending on the nature of deformation and the material properties.

Figure 7 shows the rate of deformation in the printing nip, which varies linearly with respect to the position. The rate is negative before the nip center due to a compressive deformation and positive after the nip center, as the material recovers towards to its initial thickness. The magnitude of the rate varies with the position in the nip, namely it is maximal when the paper enters or exits the nip while minimal at the center of the nip. Moreover, unlike the strain that is independent of the printing speed, the rate of the strain increases linearly with the printing speed, as shown in the figure.

\section{Nip Dynamics of Paper Substrate}

The Maxwell model may represent a paper substrate that goes through a nip defined by a pair of rigid cylinders, as it is the case of Roto Gravure or calendaring with hard nips. When going through the nip, the paper structure undergoes both elastic and plastic deformations. Due to series connections of the elastic and viscos elements, the plastic deformation will not be recovered after the printing nip.

Figure 8 shows the nip pressure profiles of five paper substrates. All the paper substrates have the same initial

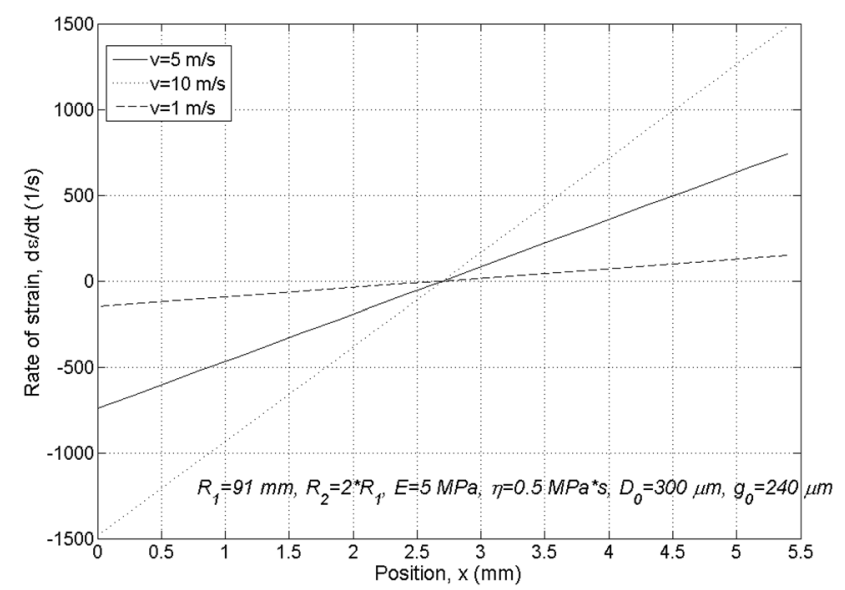

Fig. 7 The evaluation of the strain rate with respect to the position in the nip. A negative rate corresponds to increasing compressive deformation prior to the nip center, while a positive rate means recovering (reducing deformation) after the nip center 
thickness and elasticity $(E=10 \mathrm{MPa})$, but different viscosities shown in the legends in the figure. Hence, the differences in their nip pressure profiles result solely from differences in viscosity.

Obviously, the nip pressure profiles exhibit strong dependence on their viscos characteristics of the paper substrates. Due to series connection, the compressive deformation of the dash pot is responsible for plastic deformation of the whole material system (substrate). The viscosity of the dash pot indicates the resistance to the deformation. Strong viscosity implies strong resistance or small plastic deformation, while low viscosity means easy to be deformed and great plastic deformation. Hence, the peak nip pressure increases with increasing viscosity. A dashpot of infinite viscosity means that no plastic deformation or the substrate is fully elastic. Because the plastic deformation remains after the nip center, unable to fill the geometric gap between the cylinders after the nip center. This results in different effective nip widths for the substrates of different viscosity. The stronger the viscosity, the closer the effective nip width to the nominal nip width. Therefore, a paper substrate of low viscosity usually possesses bulky paper structure, will result in short nip width and low peak nip pressure. Furthermore, the nip pressure reaches its maximum prior to that the paper reaches the geometric nip center where the gap between the nip cylinders is narrowest. The shift of the peak-pressure position from the geometric nip center increases with decreasing viscosity of the material. Thus, a paper of high viscosity is more favorable from printing dynamics point of view due to longer effective nip-width and higher peak pressure. With an infinitively strong viscosity, the paper substrate becomes purely elastic and its profile becomes symmetric about the geometric nip center.

The position where the nip pressure reaches its peak equal:

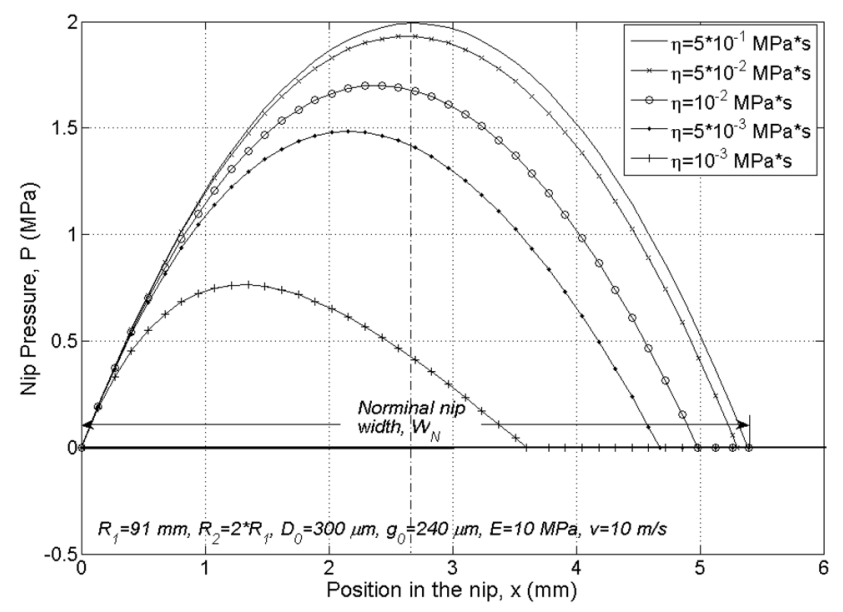

Fig. 8 Nip pressure profiles of the model paper boards which have identical properties except for viscosity shown in the legend $x_{\text {peak }}=v \cdot t_{\text {peak }}, t_{\text {peak }}=\frac{\eta}{E} \log \left(1+\frac{E}{\eta} t_{\mathrm{c}}\right)$.

These expressions indicate that, for a given printing speed, the position of peak nip pressure depends solely on the ratio of viscosity to the elasticity, $\eta / E$. Figure 9 shows explicitly the evolution of the time when the nip pressure reaches its peak value as a function of the $\eta / E$ ratio.

The effective nip width corresponds to the time, $t_{\mathrm{M}}$, when the nip pressure becomes zero after the geometric nip center. The time can be obtained from Eq. (13) by letting $\sigma(t)=0$, which fulfills the following equation:

$t_{\mathrm{M}}=\left(t_{\mathrm{c}}+\frac{\eta}{E}\right)\left[1-\exp \left(-\frac{E}{\eta} t_{\mathrm{M}}\right)\right]$.

Consequently, the effective nip width can be calculated by $W=v \times t_{\mathrm{M}}$. From these, we can once again see that the effective nip-width varies with the ratio $\eta / E$.

A paperboard is a heterogeneous material. Its properties may vary from one position to another, which may result in variations in nip pressure. For instance, two positions of a paperboard, where their Young modulus are 10 and $8 \mathrm{MPa}$, respectively. Figure 10 indicates that the heterogeneous nature of the boards may led to significant variation in the effective nip pressure.

To illustrate dependence of the nip pressure on printing speed, simulations were also made, corresponding to printing speeds, 5 and $10 \mathrm{~m} \mathrm{~s}^{-1}$, respectively. As shown in Fig. 11, a higher printing speed leads to not only stronger nip pressure (so-called speed-hardening) but also longer nip width, both promoting ink transferring. However, the effects are more prominent for boards of lower viscosity. In case of purely elastic board (viscosity equals to infinite in the Maxwell model), the nip pressure will be independent of printing

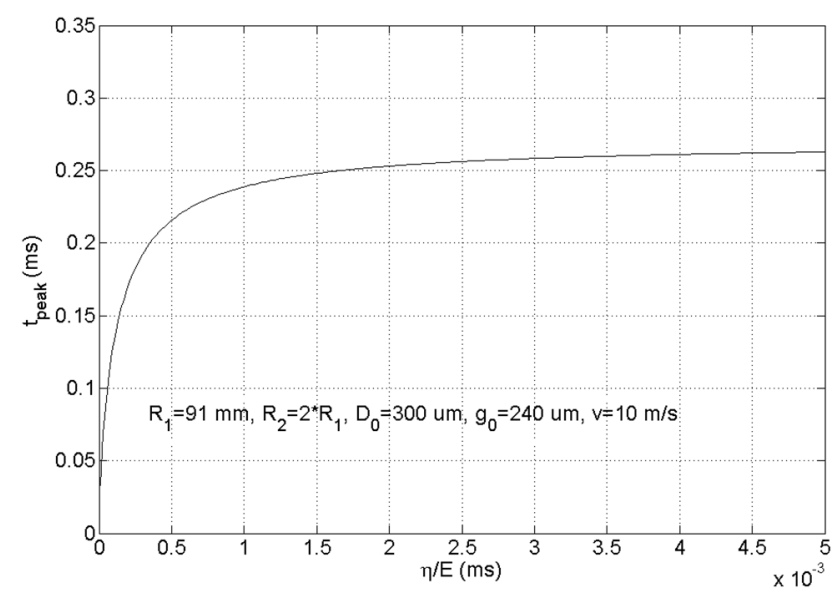

Fig. 9 Time when the nip pressure reaches its peak depends solely on the ratio $\eta / E$ ratio of the material 


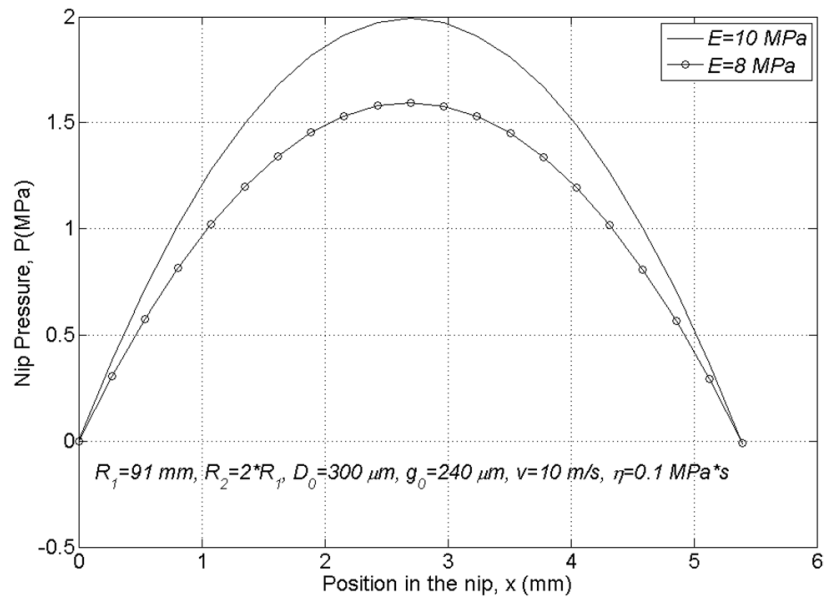

Fig. 10 Variation of the nip pressure result from the variation of the elastic Young modulus

speed. In other words, the so-called speed-hardening is a direct consequence of board viscoelasticity.

\section{Dynamic Deformation of a Print Form}

Kelvin-Voigt model may be an appropriate model to study dynamic behavior of a print form made of polymeric materials. When going through a nip defined by a pair of rigid cylinders, the print form may undergo deformations that are controlled by its elastic and viscos characteristics. However, due to parallel connections of the elastic and viscos elements, the deformation can be fully recovered with retardation after the printing nip. Yet, the time needed for a full recovery depends on the relative strengths of the elastic and viscos elements. In case of high print speed, the deformation of the print form on the nip cylinder may not have enough

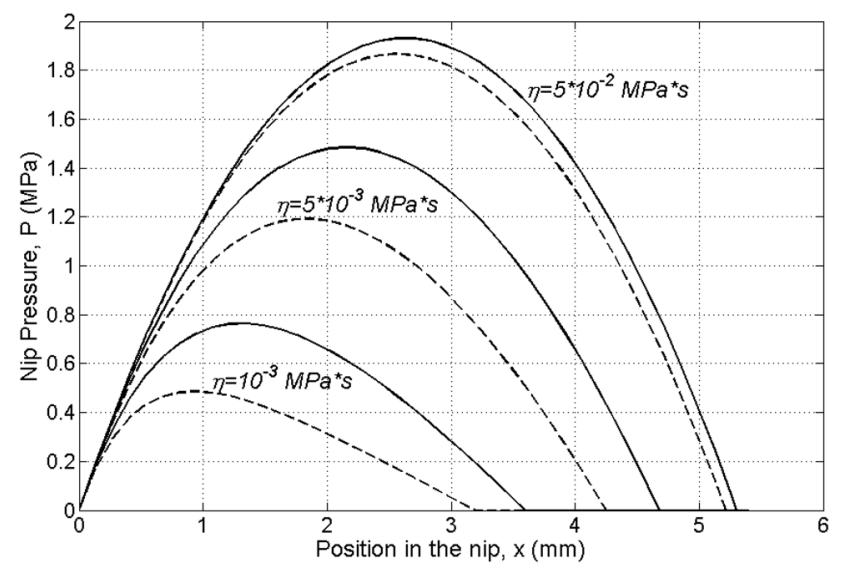

Fig. 11 Dependency of the nip pressure on printing speed with boards of different viscosities shown in the figure. The dashed lines correspond to printing speed $5 \mathrm{~m} / \mathrm{s}$, while the solid lines to $10 \mathrm{~m} \mathrm{~s}^{-1}$ time for recovering between two adjacent revolutions where the same portion of the print form engages in the printing nip.

Figure 12 depicts the nip pressure evolutions inside the print nip, with a special focus on the impact of viscos properties of the material. The compressive Young modulus was taken from the literature [18], in which rubbers of different compositions were measured. The measured values ranged from 6.2 to $7.6 \mathrm{MPa}$, and hence, a value of $E=7 \mathrm{MPa}$ was used in the simulations.

As seen, the pressure profile is symmetric when the viscosity is very low, as the elastic behavior is dominant. When the viscosity increases, the pressure profile becomes more and more asymmetric and the effective nip width decreases. Moreover, the initial nip pressure when entering the nip is non-zero; namely, there is a sudden pressure rise when entering the nip. The simulations also indicated that a strong viscosity resulted in a strong initial pressure, strong asymmetric nip-pressure profile, and shorter nip width. In other words, the nip pressure profile becomes more elastic-like when the viscos portion is low, which is in line with intuition. Furthermore, all the pressure profiles cross in the same position corresponding to the center of the print nip, because the viscos contribution or the strain rate is zero.

In a similar manner, one can obtain the position where the nip pressure reaches its peak:

$x_{\text {peak }}=v \cdot t_{\text {peak }}, t_{\text {peak }}=E\left(t_{\mathrm{c}}-\frac{\eta}{E}\right)$.

It says that the time when the nip pressure reaches its peak decreases with the ratio, $\eta / E$. Hence, the position where the position of peak nip pressure moves closer to the entering

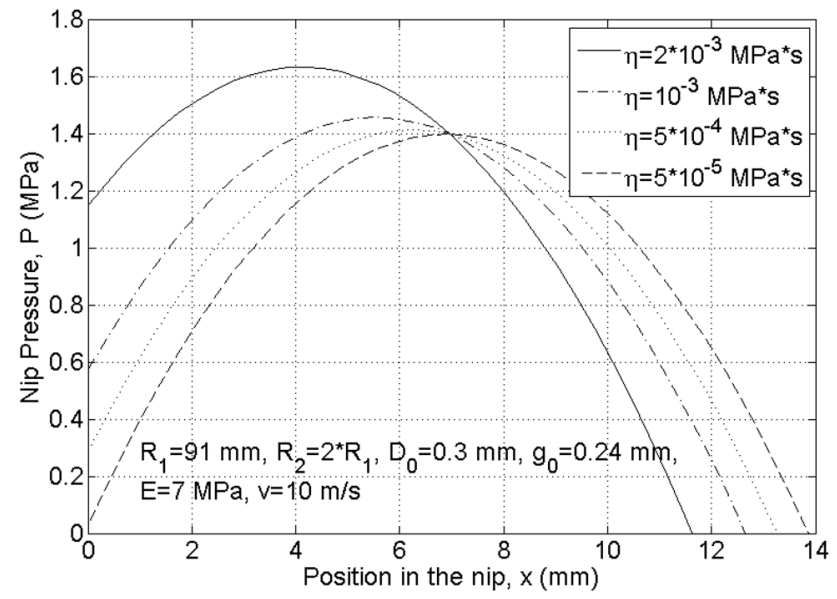

Fig. 12 Dependence of nip pressure profile with respect to viscosity of the print form. The position denoted by the arrow corresponds to the geometric nip center 
position when the viscos component increases. When the ration is equal or bigger than $t_{\mathrm{c}}$, the nip pressure reaches its peak pressure already at the entering position.

Similar to the paperboard, the print form also exhibits speed-hardening as shown in Fig. 13 in which a higher printing speed resulted in higher peak pressure. However, unlike the paper board, here the effective nip width reduced with increasing printing speed due to different connections between the elastic and the viscos components.

\section{Nip Dynamics Offset Printing}

Burgers model is a combination of Maxwell model with Kelvin-Voigt model, which has been used to simulate nip dynamics of printing processes. In the simulations, the print form, e.g., rubber blanket, was represented by the $\mathrm{K}-\mathrm{V}$ model, while the paperboard was considered as a Maxwell material. Based on the results obtained in the preceding subsections, two representative cases were studied. In case 1 , the viscos element in the $\mathrm{K}-\mathrm{V}$ model is very small, and hence, the deformation of the print form is essentially elastic. In case 2 , the viscos element in the $\mathrm{K}-\mathrm{V}$ model is strong, and hence, the deformation of the print form is highly viscoelastic.

Figure 14 depicts the nip-pressure profiles corresponding to different viscosities of the substrate materials. Since the viscosity of the print form is small, $\eta_{1}=10^{-5} \mathrm{MPa} \mathrm{s}$, the deformation of the print form is essentially elastic. Then, the viscoelasticity of the material sandwich (print form + paperboard) is dominated by the viscoelastic characteristics of the paperboard described by the Maxwell model. Indeed, the pressure profiles shown in Fig. 14 share great similarities as Fig. 8, i.e., both the peak pressure and the effective nip width increase with increasing

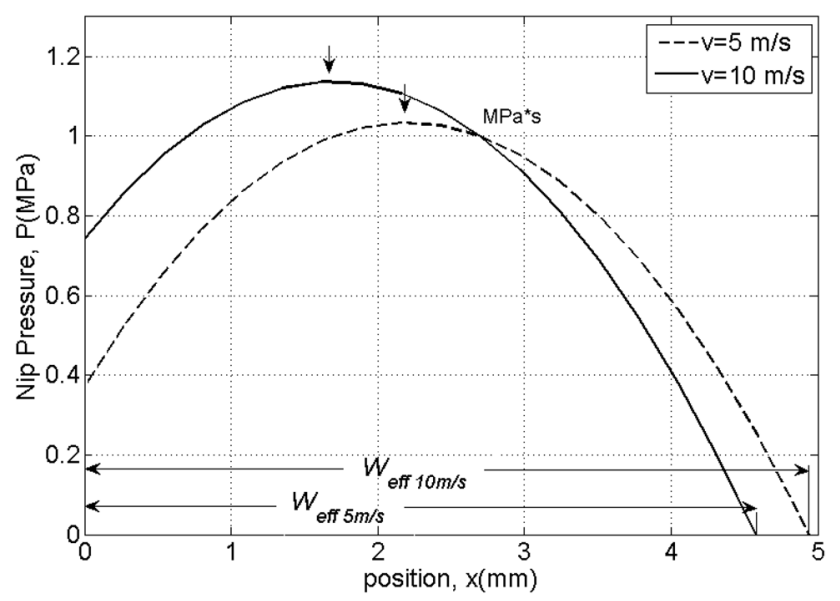

Fig. 13 Dependence of nip pressure on the printing speed for the print form, $E=7 \mathrm{MPa}, \eta=10^{-5} \mathrm{MPa}^{*} \mathrm{~s}$, the dashed line for $5 \mathrm{~m} / \mathrm{s}$, while the solid lines for $10 \mathrm{~m} \mathrm{~s}^{-1}$ viscosity and the profile tends to be symmetric when the viscosity is high. Figure 15 displays simulations of case 2 , where the viscosity of the print form is rather strong, $\eta_{1}=2 \times 10^{-2} \mathrm{MPa}^{*} \mathrm{~s}$, which led to obvious sudden rise in pressure at the nip entrance as it was shown in Fig. 12. Compared to the case 1, the peak nip pressures in Fig. 15 are higher, while their nip widths are shorter than their countparts shown in Fig. 14. Furthermore, the pressure profiles differ from case 1; for example, the profile is not symmetric even when the viscosity of the paperboard is strong. This can be attributed to the strong viscosity of the print form which results in obvious viscoelatic characteristics of the material sandwich.

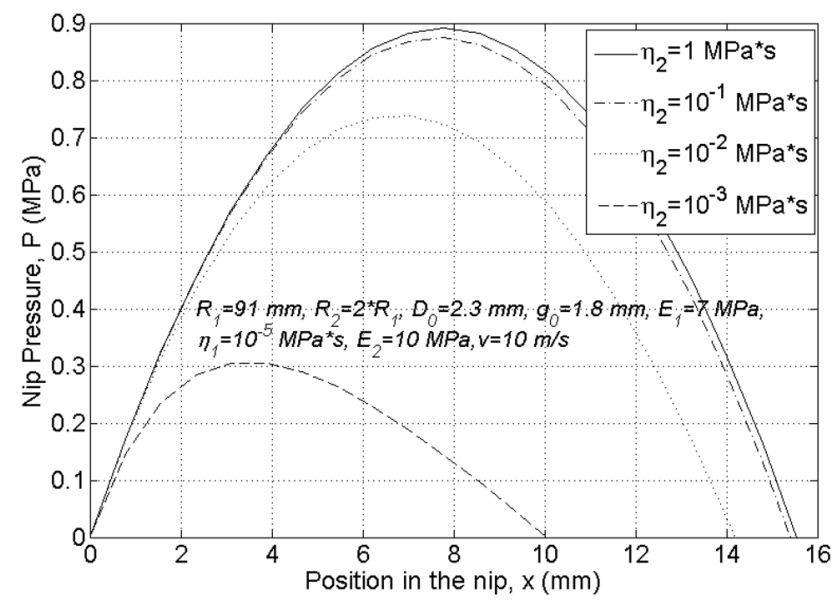

Fig. 14 Simulations with Burgers model, case 1: dependence of nip pressure profiles on the viscosity of the board $\left(\eta_{2}\right)$ shown in the legend when the viscosity of the print form $\left(\eta_{1}\right)$ is weak

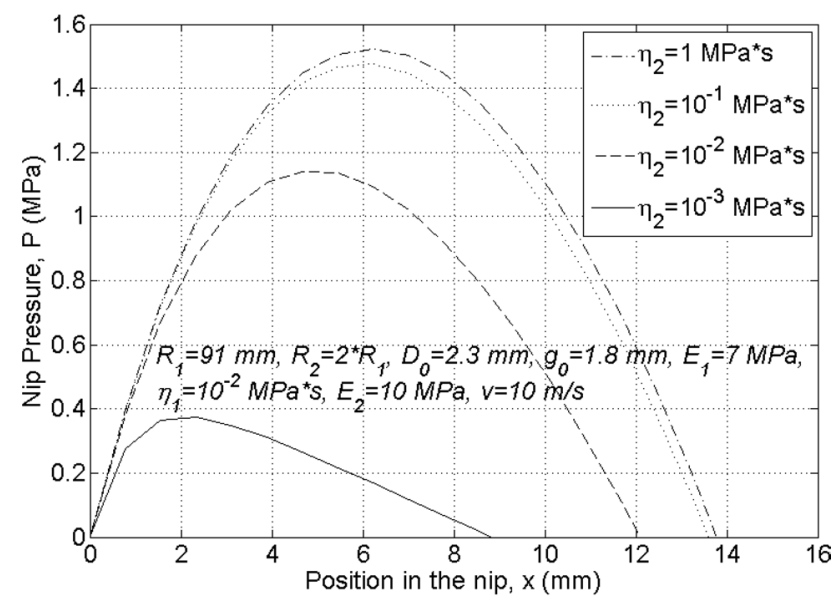

Fig. 15 Simulations with Burgers model, case 2: dependence of nip pressure profiles on the viscosity of the board $\left(\eta_{2}\right)$ shown in the legend when the viscosity of the print form, $\eta_{1}$, is strong 


\section{Discussion}

There are clear differences in dynamic behavior between a Maxwell material and a Kelvin-Voigt material. First, for a Maxwell material, the initial nip pressure when the paper entering the nip is zero and independent of the viscos component of the material. For a Kelvin-Voigt material, its initial nip is non-zero and shows a sudden rise dominated by the viscos characteristics. The initial nip pressure increases with its viscos component and rate of strain or printing speed. Second, the nip profiles of the materials also differ. For the Maxwell material (Fig. 8), when the viscos component is weak, the nip pressure file deviate strongly from symmetric shape defined by the nip geometry. The deviation reduces when the viscos component is strong. The dynamic behavior of a Kelvin-Voigt material is the opposite. As seen from Fig. 14, the deviation of the nip pressure profile from its nominal is strong when the viscos component is strong. And the deviation reduces when the viscos component is weak. Third, the effective nip width increases with increasing material viscosity for the Maxwell material while decreases for the Kelvin-Voigt material.

Both materials exhibit speed-hardening, as their nip pressures increase with increasing printing speed, as seen from Figs. 11 and 13. It worth to notice that even the effective nip width changes with printing speed. For the Maxwell material, the effective nip width increases with printing speed, while for the Kelvin-Voigt material, the effective nip width decreases with printing speed. As the Burgers material is a combination of those two, its viscoelastic behavior is more complex.

Paperboard is in variety of sandwich layers. When compressively deformed densification occurs in the $\mathrm{Z}$ direction, which leads to non-linear stress-strain relationship or the Young's modulus increases with deformation [20]. Even though the Young's modulus has been treated as constant, the proposed framework can easily be extended to situations where the Young's modulus is not constant. For instance, one may divide the total compression zone into multiple sub-regimes, and in each of the sub-regimes, the Young's modulus can be regarded as constant. Works along this line is on-going and will be published elsewhere.

\section{Conclusion}

A theoretical framework of nip dynamics of conventional printing is proposed, built upon two models. The compression model is deducted from nip geometry and printing speed, from which both the strain and the rate of the strain in the nip can be obtained. The nip dynamics model is deducted from physics laws, from which nip pressure can be obtained. In the models, paperboard and print form materials (rubber blanket, polymeric cliché, cushioning foam, etc.) are regarded as basic building blocks of the materials sandwich. The dynamic behaviors of the materials sandwich depend on the viscoelastic properties of building blocks, which are represented by elastic strings and dashpots. When going through the print nip, the materials sandwich undergoes compressive deformation before the nip center and partial recovery after the nip center. The nip pressure exerted onto the materials sandwich depends not only on the nip geometry, but also on the viscoelastic properties of the materials sandwich and printing speed.

The effects of viscoelastic characteristics of paper substrate and print form materials on the nip pressure, which become pronounced in a full-scale printing process due to high speed, are accounted and illustrated by three physical models, e.g., Maxwell model, Kelvin-Voigt model, and Burgers model. Details of the nip dynamic features, shape, amplitude, duration, and effective nip width, etc., have been worked out. The viscoelastic nature of the materials is responsible for the so-called speed-hardening, asymmetric nip profile, variations in the nip amplitude and effective nip width, etc. Moreover, how the viscoelastic properties of the materials affect the nip dynamics depends on the how the elastic components and the viscos count parts are connected with each other.

Acknowledgements Open access funding provided by RISE Research Institutes of Sweden. This work was conducted within RISE Bioeconomy Research Programme, program area Packaging. Financial supports from government founding through RISE SK medel and from industrial partners: BillerudKorsnäs, ITC, Miller Graphics, Stora Enso, Tetra Pak, UPM are acknowledged.

Open Access This article is licensed under a Creative Commons Attribution 4.0 International License, which permits use, sharing, adaptation, distribution and reproduction in any medium or format, as long as you give appropriate credit to the original author(s) and the source, provide a link to the Creative Commons licence, and indicate if changes were made. The images or other third party material in this article are included in the article's Creative Commons licence, unless indicated otherwise in a credit line to the material. If material is not included in the article's Creative Commons licence and your intended use is not permitted by statutory regulation or exceeds the permitted use, you will need to obtain permission directly from the copyright holder. To view a copy of this licence, visit http://creativecommons.org/licenses/by/4.0/.

\section{Appendix: Solution of the Burgers Model}

Burgers model combines Maxwell model with Kelvin-Voigt model, which represents a sandwich type of material system, e.g., a Maxwell material and a Kelvin-Voigt material in series. The mathematical expression for the model is: 
$A \cdot \ddot{\sigma}+B \cdot \dot{\sigma}+\sigma=D \cdot \dot{\varepsilon}+E \ddot{\varepsilon}$,

where

$A=\frac{\eta_{1} \eta_{2}}{E_{1} E_{2}}, B=\left(\frac{\eta_{1}}{E_{1}}+\frac{\eta_{2}}{E_{1}}+\frac{\eta_{2}}{E_{2}}\right), D=\eta_{2}, E=\frac{\eta_{1} \eta_{2}}{E_{1}}$.

The general solution of the Eq. (25) is:

$\sigma(t)=C_{1} \mathrm{e}^{r_{+} t}+C_{2} \mathrm{e}^{r_{-} t}+C_{3}\left(t-t_{c}\right)+C_{4}$,

where

$r_{ \pm}=\frac{-B \pm \sqrt{B^{2}-4 A}}{2 A}$.

Take into consideration of that:

$\varepsilon(t)=\varepsilon_{0}+\frac{\emptyset}{D_{0}}\left(t_{\mathrm{c}}-t\right)^{2}, \dot{\varepsilon}=2 \frac{\emptyset}{D_{0}}\left(t-t_{c}\right), \ddot{\varepsilon}=2 \frac{\emptyset}{D_{0}}$,

we obtain the following relationships:

$C_{3}\left(t-t_{c}\right)+C_{4}+B \cdot C_{3}=2 D \frac{\emptyset}{D_{0}}\left(t-t_{c}\right)+2 E \frac{\emptyset}{D_{0}}$.

Hence:

$C_{3}=2 D \frac{\emptyset}{D_{0}}$

$B \cdot C_{3}+C_{4}=2 E \frac{\emptyset}{D_{0}}$.

Hence, there are only two unknowns remain in the general solution given by Eq. (27), which can be determined by the initial conditions at $t=0$, e.g., the initial stress and its derivative. Using these initial conditions $(t=0)$, we obtain further two relationships between

$\sigma_{0}=C_{1}+C_{2}-C_{3} t_{\mathrm{c}}+C_{4}$,

and

$\dot{\sigma}_{0}=r_{+} C_{1}+r_{-} C_{2}+C_{3}$.

With considerations of the strains and stresses of the spring and the dashpot components in the Burgers model (Fig. 5), one can obtain:

$\sigma_{0}=0$,

and

$\dot{\sigma}_{0}=-2 \frac{E_{2} \cdot \emptyset}{D_{0}} t_{\mathrm{c}}$.

Thus, the initial conditions given in Eqs. (31-34) can be rewritten in a more compact form: $\left(\begin{array}{cccc}1 & 1 & -t_{\mathrm{c}} & 1 \\ r_{+} & r_{-} & 1 & 0 \\ 0 & 0 & 1 & 0 \\ 0 & 0 & B & 1\end{array}\right)\left(\begin{array}{l}C_{1} \\ C_{2} \\ C_{3} \\ C_{4}\end{array}\right)=\frac{2 \emptyset}{D_{0}}\left(\begin{array}{c}0 \\ -E_{2} t_{\mathrm{c}} \\ D \\ E\end{array}\right)$

\section{References}

1. Biermann CJ (1996) Printing and the graphic arts. In: Biermann CJ (ed) Handbook of Pulping and Papermaking. Academic Press, San Diego

2. Hsu B (1961) The influence of paper compressibility on ink penetration. Appl sci Res 10:277

3. ISO8791-4 (2017) Determination of roughness/smoothness (air leak methods) — part 4: print surface method. ISO.

4. Holmvall M (2010) NIP mechanics, hydrodynamics and print quality in flexo post-printing. Mid Sweden University

5. Holmvall M, Uesaka T (2007) Nip mechanics of flexo post-printing on corrugated board. J Compos Mater 41(17):2129-2145

6. Holmvall M, Uesaka T (2008) Striping of corrugated board in full-tone flexo post-printing. Appita J 61(1):35-40

7. Uesaka T (2012) Mechanics in printing nip for paper and board. In: Niskanen K (ed) Mechanics of paper products. Mouton de Gruyter, Berlin, pp 181-193

8. Dubé M et al (2008) Hydrodynamics of ink transfer. J Pulp Pap Sci 34:174-181

9. Jurkiewicz A, Pyryev Y (2013) Relationship in between compression of the rollers and contact area width and the clamp force. Chall Mod Technol 4(2):31-36

10. Dwan A (1987) Paper complexity and the interpretation of conservation research. J Am Conserve Res 26(1):1-17

11. Kolseth, P. and A. de Ruvo, The measurement of viscoelastic behavior for the characterization of time-temperature-and humidity-dependent properties, in Handbook of Physical and Mechanical Testing of Paper and Paper board, R.E. Mark, et al., Editors. 1983, CRC Press: New York.

12. Sharma A, Shukla A, Prosser RA (2002) Mechanical characterization of sort materials using high speed photography and split hopkinson pressure bar technique. J Mater Sci 37:1005-1027

13. Granlöf L, Yang L (2019) Techniques for static, dynamic and surface compressibility measurements of paper substrate. In: Ridgway C (ed) Advances in printing and media technology. MMXIX, Darmstadt

14. ASTM-D2221-01 (2015) Standard test method for creep properties of package cushioning materials

15. Gunderson DE (1981) A method for compressive creep testing of paperboard. Tappi 64(11):67-71

16. Köstner V et al (2018) Measuring the creep behaviour of corrugated board by cascade and individual test rig. J Appl Packag Res 10(1):46-61

17. Hussain $S$, Coffin D, Todoroki $C$ (2017) Investigating Creep in Corrugated Packaging. Packaging Technol Sci 30(12):757-770

18. Fediuc DO et al (2003) Compression modulus of elastomers. Bull Polytech Int Iasi Constr Architect Sec 61:157-166

19. Skrzypek JJ, Ganczarski AW (2015) Constitutive equations for isotropic and anisotropic linear viscoelastic materials engineering materials. In: Skrzypek AGJ (ed) Mechanics of anisotropic materials. Springer, Cham

20. Litvinov V, Farnood R (2010) Modeling of the compression of coated papers in a soft nip. J Mater Sci 45:216-226

21. Roylance D (2001) Engineering viscoelasticity. Massachusetts Institute of Technology, Cambridge

22. Sperling LH (1992) Introduction to physical polymer science, 2nd edn. Wiley Interscience, London 
23. Kolachenko A (2006) Mechanics of paper webs in printing press applications, Doctoral T. In: Solid mechanics. Royal Institute of Technology, Stockholm
Publisher's Note Springer Nature remains neutral with regard to jurisdictional claims in published maps and institutional affiliations. 